\title{
China guidelines for the diagnosis and treatment of myasthenia gravis
}

\author{
Zhu-Yi Li
}

Department of Neurology, Tangdu Hospital, The Fourth Military Medical University, Xi'an 710038, Shaanxi, China.

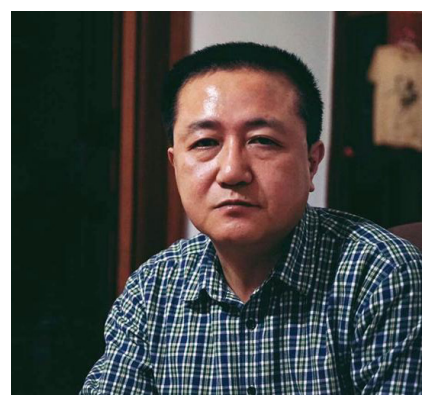

\section{Dr. Zhu-Yi Li, Professor of Neurology}

Dr. Li is the Director of Department of Neurology in Tang Du Hospital of the Fourth Military Medical University. He graduated from the Fourth Military Medical University in 1985. He has been engaged in the prevention and treatment of myasthenia gravis in Department of Neurosciences in University of Oxford and Case Western Reserve University. Dr. Li is currently the Vice Chairman of Neuroimmunology branch of Chinese Society for Immunology, Neuroimmunology Study Group of Chinese Society of Neurology, the Chairman of Chinese Society of Neurology, the Vice Chairman of Mobile Health branch of Chinese Stroke Association, Neuroimmunology Committee of Neurology branch of Chinese Medical Doctor Association, the Chairman of Shaanxi Neurology Association, the Associate EditorIn-Chief of Chinese Journal of Neuroimmunology and Neurology, and the Editorial Board Member of Chinese Journal of Neurology.

Being dedicated in researches of clinical Neurology, Dr. Li is proficient in neuroimmunology diseases such as the prevention and treatment of myasthenia gravis. Dr. Li had 9 research projects supported by Natural Science Foundation of China (NSFC), and 1 research project supported by Innovative Research Team Foundation of Ministry of Education. He has published more than 200 articles, 40 of which were published in journals indexed by SCI.

Myasthenia gravis (MG) is neuromuscular disorder induced neurotransmission defects at the neuromuscular junctions. $M G$ is an autoimmune disease in which the autologous immune system, including the corresponding antibodies, immune cells and complement systems, attacks the cholinergic receptor (AChRs) of the postsynaptic membrane, resulting in weakness of skeletal muscle. A rare portion of MG cases is mediated with antibodies specific to muscle specific kinase (MuSK) and lowdensity lipoprotein receptor related protein 4 (LRP4). The major clinical symptoms of MG are presented as weakness of skeletal muscle, fatigue prone, which are worsened after exercise. Adequate rest and treatment with cholinesterase inhibitors could significantly relieve and reduce the symptoms. The average onset rate is about $8-20$ cases per 100,000 people. ${ }^{[1]} \mathrm{MG}$ is not age specific but there is a higher onset rate in females than males at the population before 40 of age. Such

Corresponding Author: Prof. Zhu-Yi Li, Department of Neurology, Tangdu Hospital, The Fourth Military Medical University, No. 1 Xinsi Road, Xi'an 710038, Shaanxi, China. E-mail: lizhuyi@fmmu.edu.cn

\begin{tabular}{|l|l|}
\hline \multicolumn{2}{|c|}{ Access this article online } \\
\hline Quick Response Code: & Website: \\
\hline & www.nnjournal.net \\
\cline { 2 - 3 } & Dol: 10.20517/2347-8659.2015.60 \\
\hline
\end{tabular}

gender preference is reversed after 50 -year-old. At the age category of 40-50, there is no gender preference.

\section{CLINICAL SYMPTOMS AND CLASSIFICATIONS}

\section{Clinical symptoms}

Systemic skeletal muscle may be affected. However, at the early stage of MG, there may be firstly the muscle weakness of extraocular, throat and limbs. Seemingly, the skeletal muscles innervated by the cranial nerves are more susceptible than those innervated by spinal nerves. The weakness of skeletal muscles initiates at a group of muscles first and gradually spreads to other groups of muscles and eventually the whole system. For some patients of MG, they may rapidly experience weakness of all skeletal muscles and even myasthenic crisis.

The skeletal muscle weakness of MG is volatile with ease of fatigue, which are worsening towards the end of the day. The weakness and fatigability is progressively worsening during physical activity,

This is an open access article distributed under the terms of the Creative Commons Attribution-NonCommercial-ShareAlike 3.0 License, which allows others to remix, tweak, and build upon the work non-commercially, as long as the author is credited and the new creations are licensed under the identical terms.

For reprints contact: service@oaepublish.com

Cite this article as: Li ZY. China guidelines for the diagnosis and treatment of myasthenia gravis. Neuroimmunol Neuroinflammation 2016;3:1-9.

Received: 03-12-2015; Accepted: 05-01-2016 
which is improved after adequate rest. The initial symptom is the ptosis either symmetrically or asymmetrically and diplopia which are observed in more than $80 \%$ of patients. ${ }^{[2]}$ In certain cases, alternative or bilaterial ptosis and nystagmus are also observed. Patients suffer also from lagophthalmos. However, pupil size remains normal.

Due to the weakness of the muscles involved in swallowing, there is dysphagia, difficulty of chewing and velopharyngeal insufficiency. For the symptoms of vocality, patients of MG have dysarthria and hypophonia, some of who may also have nasality. Weakness of facial muscle leads to hanging jaw sign, shallow nasolabial fold, leaky cheek blowing, snarling expression when smiling and sleepy or sad expression. Weakness of cervical muscle, patients could not hold their upright easily. Although it is usually not the primary symptoms of MG, limb movement is highly affected spreading from the proximal ends. In certain cases, the respiratory muscle is under myasthenic crisis which leads to dyspnea. Assisted ventilation is necessarily needed to sustain life..$^{[3,4,5]}$

\section{Classification: modified Osserman scale}

Class I: ptosis and diplopia without other muscle weakness elsewhere for 2 years.

Class II: generalized symptoms with more than one set of weak muscle: (1) mild generalized form; Weakness in limbs with or without ocular signs, but without prominent bulbar signs. Patients could live independently; (2) moderate generalized or faciopharyngeal form; symptoms as in II A but with bulbar signs. Patients could live independently.

Class III: severe acute generalized form; acute onset and rapid development. Faciopharyngeal symptoms were observed in the first few weeks to months followed by respiratory insufficiency with or without ocular signs. Patients could live independently.

Class IV: severe chronic generalized form; unapparent signs at early stage due to the slow pathological progress. All symptoms stated in Class I, II and III are developing within 2 years.

Class V: muscle atrophy form; severe development with also muscle atrophy within 6 months.

\section{EXAMINATION}

Methyl sulfate neostigmine test

Intramuscular administration of 1.0-1.5 mg methyl sulfate neostigmine for adult. Atropine $(0.5 \mathrm{mg})$ could be applied intramuscularly if there are any muscarinic cholinergic receptor-mediated side effects. For children, neostigmine should be reduced to $0.02-0.03 \mathrm{mg} / \mathrm{kg}$, while total amount should not be more than $1.0 \mathrm{mg}$. Test should be performed at the muscle with significant symptoms and referred to the clinical absolute score for MG. The baseline of muscle tone should be firstly recorded, which is repeated every $10 \mathrm{~min}$ for $1 \mathrm{~h}$. The relative score, as the diagnostic value, should hence be pooled by the absolute score of the trial with most significant improvement according to the following formula:

Relative score $=$ (Baseline-absolute score of each trial)/Baseline $\times 100 \%$

There is usually less than $25 \%$ of negative diagnosis while more than $60 \%$ for positive diagnosis, and $25-60 \%$ are as suspicious positive cases. Further tests are needed to confirm the negative diagnosis.

\section{Electromyography}

\section{Repetitive nerve stimulation}

Repetitive nerve stimulation (RNS) is an electrical stimulation to nerves, such as facial, accessory, axillary and ulnar nerves, for MG diagnosis, with repetitive and high-powered low frequency $(2-5 \mathrm{~Hz})$ signal. Compound muscle action potential (CMAP) over the testing muscles will be recorded.

The duration of stimulation is about $3 \mathrm{~s}$. The decrement of CMAP of MG is measured by comparing the CMAP value of the fourth or fifth stimuli to that of the first stimulus. Diagnosis will be concluded as positive when there is more than $10 \%$ reduction. MG patients on acetylcholinesterase inhibitor medication should not receive this test until 12-18 h of washing out. For diagnosing presynaptic lesions, frequency of RNS should be increased to 10-20 Hz. Increment with more than $100 \%$ should be classified as abnormal.

\section{Single fiber $E M G$}

Single fiber EMG (SFEMG) is to measure the variable latency of the single axon innervation to the muscle fibers, known as Jitter. The variable latency is usually about 15-35 s, of which more than 55 ms will be classified as increased variability of latency. Two or more variable latency in every 20 Jitters of a single set of muscle will be classified as abnormal. Any block during SFEMG should also be classified as abnormal. Despite of the significant sensitivity, SFEMG is not specific for MG, which is mainly for Class I MG and those cases without positive outcomes in RNS test. Furthermore, SFEMG is not affected by any acetylcholinesterase inhibitors. 
Serum antibody tests

$A C h R$

For diagnosing MG with the specific antibodies, positive results could be detected in about 50-60\% patients with ptosis and other ocular sign, while about $85-90 \%$ patients with generalized sign would have positive outcomes. Based on also the medical history of muscle weakness, positive diagnosis is enough to confirm MG. Further tests are needed if it is a negative outcome.

\section{Anti-MuSK}

Anti-MuSK could be detected in some patients with generalized sign of muscle weakness who have negative outcomes in the test of AChR antibody, while other patients may present positive outcomes of lowdensity LRP-4 antibody or other antibodies without known antigens at the neuromuscular junctions. Negative outcomes could be due to the low levels or weak affinity of these antibodies, resulting in undetectable outcome. It should also be noted that the ratio of anti-MuSK positive result in Western population is usually higher that that in Asian population.

\section{Anti-striated muscle antibodies}

Anti-striated muscle antibodies include anti-titin, anti-RyR and etc. This type of antibodies is usually detected in those MG patients with severe signs, desensitization of conventional therapies of MG and with also thymoma. This test is not for MG diagnosis but it strong indicates the opportunity of thymoma and other related transformations.

\section{Thymus imaging}

There are approximately 20-25\% MG patients suffer also from thymic tumors while $80 \%$ have abnormal condition of thymus. Amongst the MG patients with thymic tumors, $20-25 \%$ of them present MG symptoms. ${ }^{[7]}$ Ninety-four percent thymic tumors could be positive diagnosed by mediastinal CT. However, some cases are only diagnosed by advanced CT scan or MRIs.

\section{DIAGNOSIS}

Bases of diagnosis

\section{Clinical symptoms}

There is patchy distribution of weakness on certain particular striated muscle, exhibiting volatility and ease of fatigue. These MG symptoms are usually worsened across the day and after activities, which would be relieved after rest. In majority, ocular muscle is the common and first victim.

\section{Pharmacological response}

Positive outcome in neostigmine test.

\section{EMG tests}

Ten percent decrement of CMAP and 2 or more variable latency ( $>55 \mathrm{~ms}$ ) in SFEMG with or without any blocks.

\section{Antibody tests}

Test of anti-AChR in blood sample of generalized form of MG is positive. Anti-MuSK and LRP-4 would only be detected in some rare cases of MG.

With all clinical symptoms, and positive outcomes of pharmacological and electrophysiological tests, it can be diagnosed as MG. When it is available, blood test of anti-AChR or other related antibodies could be adopted for further confirmation. Furthermore, other unknown diseases may interfere the diagnosis.

\section{Differential diagnoses}

\section{Ocular $M G$}

Miller-Fisher syndrome: it is a derivative of GuillainBarré syndrome, which is with acute paralysis of extraocular muscle, ataxia and loss of tendon reflexes. EMG results indicate there is delay of neurotransmission. Furthermore, in the analysis of cerebrospinal fluid, there is protein-cell separation. In some cases, GQ1b antibody is detectable.

Chronic progressive external ophthalmoplegia (CPEO): $\mathrm{CPEO}$ is one of the mitochondrial myopathies, patients of which experienced symmetrical, bilateral and progressive ptosis, paralysis of extraocular muscle, myogenic lesions and lactic acidosis. Some patients may experience also weakness of proximal limbs and delay of peripheral nerve transmission. Muscle biopsy and gene tests are needed for further confirmation.

Oculopharyngeal muscular dystrophy (OPMD): OPMD is a progressive muscular dystrophy, the patients of which experience progressive ptosis and weakness of the extraocular muscles. There is also a slight elevation of the serum level of creatine kinase. EMG diagnosis of OPMD shows myogenic lesion. Muscle biopsy and gene tests are needed for further confirmation. 
Orbital Lesions: this condition could be due to orbital tumors, abscess and inflammatory pseudotumors. Patients experienced paralysis of extraocular muscle, conjunctival hyperemia, exophthalmos and edema in eyelids. This could be confirmed by orbital MRI, CT and ultrasonic scans.

Graves' disease: this is a thyroid-related autoimmune disease, patients of which experience hyperthyroidism or hypothyroidism, eyelid lag and weakness of extraocular muscle. In orbital CT scan, extraocular muscle is swelling. There is positive detection of TSH receptor antibody, namely TRAb.

Meige syndrome: this is an extrapyramidal disorder, patients of which experience unilateral or bilateral blepharospasm, reduction of eye fission, non-rhythmic tonic spasms at face, jaw and tongue. Dopamine receptors antagonists or local administration of type A botulinum toxin can improve these symptoms.

\section{Generalized MG}

Guillain-Barré syndrome: this is an immunemediated acute inflammatory peripheral neuropathy, patients of which experience flaccid and weakness of limbs, reduction or loss of tendon reflexes. By EMG, the motor neuronal function presents as increment of conduction latency, slower innervation velocity, blockade and discrete and abnormal waveform.

Chronic inflammatory demyelinating polyneuropathy (CIDP): CIDP is an immune-mediated disorder of the peripheral nervous system, the patients of which experience flaccid and weakness of extremities, hypoesthesia, reduction or loss of deep tendon reflexes. There is a reduction of conduction velocities in motor and sensory neurons, abnormal and block waveform. There is also protein-cell separation in CSF. Diagnosis could be confirmed by biopsy of PNS tissue.

Lambert-Eaton syndrome: this is an autoimmune disorder mediated with the antibodies attacking the presynaptic voltage-gated calcium channels, the patients of which experience muscle weakness and fatigue of proximal limbs, muscle tone enhancement after brief activity but weakness after sustain activities, autonomic nervous system sign, such as dry mouth, orthostatic hypotension, slow gastrointestinal motility, pupil dilation, etc. In EMG test, low frequency repetitive stimulation induces small amplitudes of CMAP but increased amplitudes in high frequency repetitive stimulation. This disorder commonly happens with certain malignancies, particularly small cell lung cancer.

Progressive spinal muscular atrophy (PSMA): PSMA is a rare type of motor neuron disease, patients of which experience flaccid, weakness and atrophy of extremities, muscle fasciculations, reduction or loss of deep tendon reflexes. EMG result shows that there is denervation. At resting stage, there is fibrillating potentials and positive peak waves, and even fasciculation potentials in certain cases. Duration of potentials of motor units is broaden, volatility and number of multiphase wave increase during mild muscle contraction. However, the potentials decrease in amplitude and present as single or mixed phases during maximal contraction. Moreover, the conduction velocity of sensory neuron is normal.

Polymyositis: this is a multifactorial inflammation in interstitial area of skeletal muscle, patients of which experience progressive flaccid and weakness of muscle and pain. EMG indicates myogenic lesion and cardial level of creatine kinase is significantly elevated. This disorder could be confirmed by biopsy, which is curable by corticosteroids.

Botulism: botulinum toxin damages the presynaptic membrane of neuromuscular junctions, leading to extraocular muscle paralysis, dilation of pupil but retarded light reflex, weaknesses in swallowing and chewing, dysarthria and symmetrical flaccid paralysis of limbs. If respiratory muscles are affected, there could be Lambert-Eaton myasthenic syndrome liked autonomous signs. There is no significant decrement of EMG in the low frequency repetitive nerve stimulation. However, there is increment of amplitude or no changes in high frequency repetitive nerve stimulation, which is dependent on the severity of poisoning. Diagnosis could be confirmed by isolating and identifying the botulinum toxin in the consumed food.

Metabolic myopathy: This is a disorder led by the compromised muscle metabolism, lipid metabolism or lesion of mitochrondria, patients of which experience flaccid and weakness of limbs and fatigue, reduction or loss of deep tendon reflexes. There is myogenic lesion in EMG and normal or slight elevation of cardiac enzymes levels. Diagnosis could be confirmed by muscle biopsy and gene tests.

\section{TREATMENT OF MG}

Therapeutic approaches

\section{Cholinesterase inhibitors}

Such inhibitors are the first-line drugs for $\mathrm{MG}$ 
treatment, which mainly ameliorate the clinical symptoms, particularly for the initial treatment of newly diagnosed patients of MG and as a single agent for long-term treatment of cases of mild MG ${ }^{[8]}$ although it is not recommended. Dose should be individualized and combined with other immunosuppressive drugs. Pyridostigmine bromide is the most commonly used cholinesterase inhibitor for MG treatment. Side effects include nausea, diarrhea, stomach cramp, bradycardia and increase of oral and respiratory secretions. In China, the maximum oral dosage per day is $480 \mathrm{mg}$ and three to four times.

\section{Immunosuppressive drugs}

Glucocorticoids: it is a potent anti-inflammatory and immunosuppressive agent, efficient in MG treatment with significant improvement in $70-80 \%$ cases. ${ }^{[9]}$ The commonly used glucocorticoids for MG treatment include prednisone, methylprednisolone and dexamethasone. The indications are as followed: 0.5$1.0 \mathrm{mg} / \mathrm{kg} /$ day or $20 \mathrm{mg} /$ day prednisone at morning. According to the glucocorticoid dose conversion, 5.0 mg prednisone is equal to $4 \mathrm{mg}$ methylprednisolone and $0.75 \mathrm{mg}$ dexamethasone. Dosage should be increased by $5.0 \mathrm{mg}$ every three days till $60-80 \mathrm{mg}$. Improvement could be observed in 2 weeks, which will be significant at $6-8$ weeks after treatment. For severe cases, with the adequate communication between physician and patients, patients can receive corticosteroid therapy under mechanical ventilation. Corticosteroid therapy will be achieved by continuous intravenous perfusion of 1,000 mg/ day methylprednisolone for 3 days. Afterward, dose should be decreased to $500 \mathrm{mg} /$ day for 2 days. During this, methylprednisolone could be replaced by dexamethasone (10-20 mg/day) for 1 week. After corticosteroid therapy, patients should take prednisone or methylprednisolone at morning as aforementioned. Dosage of prednisone and methylprednisolone should be fine adjusted or individualized according to patients' conditions. If MG conditions are improved, dosage could be gradually reduced after 4-16 weeks. Typically, prednisone could be reduced by 5-10 mg every 2-4 weeks, and then 5 mg every 4-8 weeks when dose is or lower than $20 \mathrm{mg}$. According to different cases, patients could receive the lowest optimal dose every other day. Too vigorous reduction of drug will worsen the MG conditions.

In adult generalized $\mathrm{MG}$ and certain ocular $\mathrm{MG}$ cases, glucocorticoids should be reduced or terminated if there are any fluctuation or aggravation. In order to provide an optimal therapy, it is recommended to co-administer other immunosuppressants, such as azathioprine, cyclosporine A or tacrolimus.
Methylprednisolone has a more rapid therapeutic effect in MG treatment than prednisone for 1.25 fold, as the former needs not to be activated in liver. Furthermore, methylprednisolone has a higher immuosuppressive effect (18 fold) than prednisone since the former has a higher affinity to corresponding receptors. Such property produces lesser side effect and steady concentration, which is more suitable for those MG patients with compromised hepatic function.

Intensive monitoring is necessary for treatment with glucocorticoids. There are approximately 40-50\% MG patients experiencing transient aggravation and possibly myasthenic crisis. Thus, extra cautions are used for those with severe symptoms and higher risk of myasthenic crisis. It is also highly recommended to administer calcium and bisphosphonates agents for preventing osteoporosis and antacid drugs for preventing gastrointestinal complications. There is also chance to develop steroid myopathy. Chronic usage of glucocorticoids will increase appetite, body weight and central obesity, hypertension, high blood glucose, cataract, glaucoma, endocrine disorders, mental disorders, osteoporosis, osteonecrosis and other alimentary disorders.

Azathioprine: this is the first line agent for MG for both ocular and generalized forms. Azathioprine could also be co-prescribed with glucocortioid so that in short term, the dosage of glucocorticoid could be reduced. At the initial stage of treatment, glucocorticoid and azathioprine provide a better treatment than single use of glucocorticoid or azathioprine. For MG patients who are older than 3 year-old and at teenages, azathioprine could be co-prescribed when therapies with cholinesterase inhibitors and glucocorticoid are not desirable. Azathioprine should be administered in a low dose and gradually increased as this agent could elevate the hepatic enzyme activities and inhibit the marrow function. Effect will be seen 3 to 6 months after administration while the peak effect will reach after 1 to 2 years. About 70-90\% MG patients are significantly improved after this treatment.

Instruction is as followed: $1-2 \mathrm{mg} / \mathrm{kg} /$ day for children and 2-3 mg/kg/day for adult; spilted into 2-3 times oral dose per day. Chronic usage is allowed until adverse effect and intelarance are seen. About 7-10 days after azathioprine administration, blood test and hepatic functions of patients should be monitored. Side effects includes specific flu symptoms, reduciton of white blood cells and plalete, alimentary symptoms, eaken hepatic function and loss of hair. Chronic users of azathioprine should take blood test every 2 weeks, tests of hepatic and renal functions every 4 weeks. If possible, gene screening of purine methyltransferase 
deficient should be performed to reduce the risk of irrevesible marrow lesion.

Cyclosporin A: this is an immunosuppressant for generalized and ocular MG, which starts to effect 3-6 months after administration. This is mainly used when glucocorticoid and azathioprine are not effective. Cyclosporin A could also be used with glucocorticoid for improving the MG with a decreasing blood level of AchR antibody. Cyclosporin A could be used in long term with a similar effect as azathioprine but with lesser side effect. Usually cyclosporin A was taken in orally in $2-4 \mathrm{mg} / \mathrm{kg} /$ day. The blood level of cyclosporin A was monitored in order to adjust the dose. The main side effect includes hypertension, tremor, renal dysfunction, muscle ache, gingival hyperplasia and flu-like symptoms. Blood test, hepatic and renal functions are regularly checked every month.

Tacrolimus (FK-506): This is also a potent immunosuppressant used for those patients not susceptible to glucocorticoid and other immunosuppressant, particularly those RyR antibody positive. This is also used with glucocorticoid at the early stage of treatment to reduce the usage of glucocorticoid and corresponding side effect. The therapeutic effect of FK-506 onset rapidly and significant effect should be observed around 2 weeks after administration. FK-506 is usually prescribed at $3.0 \mathrm{mg} /$ day for oral dose. Blood level of FK-506 should be monitored in order to adjust the dose. MG patients with rapid metabolism should receive large dose till a significant effect observed. Side effects include alimentary symptoms, numbness, tremor, headache, hypertension, hyperglycemia, hyperkalemia, hypomagnesimia and renal failure. If no significant side effect, FK-506 could be chronically used. Blood glucose, hepatic and renal functions should be monitored every month.

Cyclophosphamide: this agent will be used when other immunosuppressants fail to provide any therapeutic effect on to severe cases MG or MG with thymus tumor. Cyclophosphamide should also be administered with glucocorticoid, which could be reduced in dose 6-12 months after treatment started. For adult, 400-800 mg cyclophosphamide per week should be intravenously perfused. Patients could also receive $100 \mathrm{mg} /$ day in two oral doses till the total amount reaches 10-20 g (for some cases, patients need $30 \mathrm{~g}$ ). For children, 3-5 $\mathrm{mg} / \mathrm{kg} /$ day (totally amount should not exceed $100 \mathrm{mg}$ ) in two oral doses was administered. When there is improvement of symptoms, dose could be reduced to $2 \mathrm{mg} / \mathrm{kg} /$ day. Extra caution should be paid for children patient receiving cyclophosphamide. Side effects includes reduction of white blood cells count, loss of hair, nausea, vomit, diarrhea, hemorrhagic cystitis and long term suppression of bone marrow mediated cancer risk.

Mycophenolate mofetil (MMF): MMF is not the first line agents for $\mathrm{MG}$ but it is also used with glucocorticoid at the early stage of treatment but not azathioprine. Usually, it will be administered at rate of $0.5-1 \mathrm{~g}$ and twice a day. When compared to azathioprine and cyclosporine, MMF is safer and with less hepatic and renal side effect. Common side effect includes alimentary symptoms, nausea, vomit and diarrhea. For MG patients taken MMF, their whole blood count will be performed once a week at the first month, twice a month at the second and third months and once a month after 3 months. If there is a reduction of neutrophils, patients should stop taking any $\mathrm{MMF}$.

Anti-human CD20 monoclonal antibody (Rituximab): Rituximab is proven to be effective in treatment of autoimmune diseases. ${ }^{[10]}$ In treatment of MG, rituximab is suitable for patients, particularly those with MuSK positive, who have no significant improvement in treatment of glucocorticoid and traditional immunosuppressants. As single agent of MG treatment, recommended dose for adult is in rate of $375 \mathrm{mg} / \mathrm{m}^{2}$ (i.v.) once a week. The treatment course is 22 days and agent is totally administered for 4 times.

Treatment with rituximab should be performed with facility of resuscitation. When there are any respiratory symptoms or hypotension, patients receiving this treatment should be monitored for 24 hours. Treatment has to be terminated when there are any adverse effects such as dyspnea, bronchospasm and hypoxemia. Other side effects include fever, chills, bronchospasm, leukopenia, thrombocytopenia and progressive multifocal leukoencephalopathy. It is also crucial to monitor any syndromes of cytokines release.

During this treatment, hepatic and renal functions, blood and urine biochemistries have to be monitored regularly. Treatment should be immediately terminated if there is any immunosuppression mediated side effects. For patients with HBsAg positive and compromised hepatic function, nucleotides (NAs) should be administered 2-4 weeks before treatment.

\section{Intravenous administration of g-globulin}

This is for acute situation and pre-operative treatment for MG patients, usually combined with immunosuppressants and glucocorticoid. ${ }^{[11]}$ g-globulin will be intravenously perfused at rate of 
$400 \mathrm{mg} / \mathrm{kg} /$ day for 5 days. Effect of this treatment will onset in 5-10 days and last for 2 months. As similar as the plasmapheresis discussed below, side effects are less but both procedures cannot be combined. For moderate and severe MG patients, repetitive treatment of this could not maximize the therapeutic effect. Side effects include headache, aceptic meningistis, flu signs and renal dysfunction.

\section{Plasmapheresis}

This is mainly for acute cases of MG, myathenic crisis and pre-operative treatment for thymectomy. ${ }^{[12]}$ This is also used for cases without further improvement after chronic treatment with immunosuppressants. Plasmapheresis should be performed every other day in the first week, totally 3 times. If there is no significant improvement, procedure should be continued once a week for 5-7 weeks. Each treatment introduces $1,500 \mathrm{~mL}$ health human plasma and $500 \mathrm{~mL} 706$ supplement. Significant effect will onset 2 days after the first or second treatment lasting for 1-2 months. Side effects include hypotension, low blood calcium, infection and hemorrhage. Plasmapheresis should be performed in aseptic environment. Termination should be used if there is any complication. MG patients with infection and receiving perfusion of g-globulin should not receive this procedure.

\section{Thymectomy}

It is crucial to perform thymectomy for the MG patients with thymus tumor, which could eliminate risks of invasion and proliferation. ${ }^{[7]}$ Thymectomy could also improve the MG signs of patients. However, in certain cases, the MG condition would be worsened. For mild MG (Osserman class I), thymectomy could not have any improvement. However, for Osserman class II to IV, particularly those with AChR antibody positive, thymectomy provides a significant improvement. MG signs would be usually reduced 2-24 months after the operation and medication could be also reduced. Although some MG patients will recover totally after thymectomy, some will experience MG reoccurrence in a few years. Generally thymectomy is beneficial for MG with abnormal thymus glands. Such operation is suitable for patients older than 18 year-old. For severe cases with non-magliant thymus tumor, treatments, such as perfusion of g-globulin, will be firstly recommended than surgery when MG signs have been slightly improved, which could also prevent post-operation myathenic crisis.

\section{Thymus radiotherapy}

The sophistication of radiological techniques makes

\begin{tabular}{lll}
$\begin{array}{l}\text { Table I: Differential diagnoses of myasthenic and } \\
\text { cholinergic crises }\end{array}$ \\
\hline \multicolumn{3}{c}{ Myasthenic crisis } \\
\hline Heart rate & Tachycardia & Bradycardia \\
Muscle & Weak & Weak and fasciculation \\
Pupil & Normal or dilated & Constricted \\
Skin & Faint and cold & Warm and flushing \\
Secretion & Normal & Increase \\
Neostigmine test & MG improved & MG aggravated weakness \\
\hline MG: myasthenia gravis &
\end{tabular}

this as a popular therapy for MG. ${ }^{[13]}$ This approach is suitable for those MG patients who experience invasive thymus hyperplasia, reoccurrence of MG and not sensitive to other medication. Daily treatment dose is 1 to $2 \mathrm{~Gy}$ and 5 times per week. The total amount is $50-60 \mathrm{~Gy}$.

\section{Others}

Respiratory muscle training and other strength training in mild case of MG could improve the muscle strength. It is highly recommended that patients should control weight and limit the daytime activity. Seasonal flu shot is also beneficial in therapy.

\section{Therapies for different types of MG}

Ocular MG: although it is more prevalent in children under 10 year old and adult above 40, this could be seen in any age groups. 80\% patients of MG experience first with ocular MG which could be controlled by individualized doses of AChE inhibitors. For better treatment, AChE inhibitors could be combined with glucocorticoid and methylprednisolone. In recent review literatures, oral dose of glucocorticoid, e.g. prednisone, is better in treat of ocular MG than only AChE inhibitors and more effective in preventing the transformation to generalized forms of MG. However, randomized and blinded clinical trials are needed to confirm this. In order to have better treatment, it is also recommended to apply immunosuppressants and glucocorticoid. Thus, glucocorticoid induced side effect could be reduced.

Generalized form: as AChE inhibitors are not effective enough to control the MG symptoms, treatment should combine with glucocorticoid and other immunosuppressants, e.g. azathioprine, cyclosporine, tacrolimus and MMF. Some cases of generalized MG need methylprednisolone, $40-50 \%$ of which may be worsened during treatment and needed endotracheal intubation or tracheotomy. High dose of g-globulin could be used when methylprednisolone fails to provide any effect. Thymectomy should be performed early for those with abnormalities of thymus glands, such as thymus tumor and thymus hyperplasia. 
Medication could usually be reduced after operation. For some cases, no more MG signs will be seen postoperation. For children, AChE inhibitors, glucocorticoid and g-globulin are beneficial for generalized form of MG. Otherwise, with cautions, patients could be treated by immunosuppressants and thymectomy.

MG crisis: ${ }^{[14,15]}$ it is the compromised respiratory muscle leading to severe difficulty in breathing, which has to be supported by artificial respiration, such as positive pressure respiration, endotreacheal intubation and tracheotomy, and monitoring the oxygen saturation and partial pressure of carbon dioxide. MG crisis could be classified as in Table 1. For myasthenic crisis, dose of AChE could be increased within the safe window till there is any improvement. Overdose of AChE could be reversed by atropine or methylprednisolone. For some cases, it is also practical to apply high dose of g-globulin and plasmapheresis. For cholinergic crisis, treatment with AChE inhibitors should be reduced or terminated and should not resume and increase gradually until 5-7 days. Atropine or combined with methylprednisolone, plasmapheresis and g-globulin could also be adopted. Nowadays, AChE inhibitors should be limited at not more than $480 \mathrm{mg}$ per day. Thus, cholinergic crisis is uncommon. If respiratory failure is found in blood gas analysis (in both type I and II), endotreacheal intubation and positive pressure respiration should be immediately applied. Artificial respiration of MG patients should have extra care to prevent lung infection and adjustment of the auxiliary breathing mode for earlier independent breathing.

MG at pregnancy: ${ }^{[16]}$ it is still not very clear that how pregnancy affects MG. For most cases, pregnancy will not aggravate MG and affect the labor time and route. AChE inhibitors and glucocorticoid are relatively safe for fetuses but other immunosuppressants may affect the embryonic development which should be terminated if pregnant. Teratogenic drugs, e.g. methotrexate and MMF, should not be used. It is also recommended for MG patients to take caution of contraception.

MG with MuSK antibody positive: generally, AChE, glucocorticoid and immunosuppressants are not effective for MG with AChR antibody negative but MuSK positive. Up to date, there is no special and effective treatment for this type of MG. plasmapheresis could relieve the MG signs for short term. There is a case report that anti-CD20 monoclonal antibody is therapeutic potent to this type of MG. ${ }^{[9]}$ Multiple thymectomy is also beneficial for this type of MG.

MG with other complications

Some MG patients could suffer from also other disorders, such as Graves diseases, polymyositis, multiple sclerosis, Sjogren's syndrome, periodic paralysis, Hashimoto 's disease, rheumatoid arthritis, systemic lupus erythematosus, Guillain-Barré syndrome, aplastic anemia. In some MG cases, cardiac muscle is also the victim, presenting abnormal EEG and arrhymia. Therefore, it is recommended to pay also attention to such condtions other than MG.

\section{Precautions for MG treatment}

There are certain contraindication for MG patients, including steroids, antibiotics (e.g. Aminoglycoside), antifungal drugs (e.g. amphotericin), cardiovascular drugs (e.g. lidocaine, quinidine, $\beta$-blockers, verapamil and etc.), antiepileptic drugs (e.g. Phenytoin, ethosuximide), antipsychotics (e.g. chlorpromazine, lithium carbonate, diazepam, clonazepam), anesthesia (e.g. morphine and meperidine) and anti-rheumatic drugs (e.g. penicillamine and chloroquine).

It is also not recommended to do soapsuds enema. Plenty of rest, staying warm, steady emotion are also important for recovery from MG.

\section{Prognosis}

Ten-twenty percent of MG patients in ocular form will spontaneously heal, while $20-30 \%$ only experience extraocular MG. For the rest, more than 85\% will gradually spread the signs to medulla oblongata and skeletal muscle, developing generalized form in 3 years. The pathogenesis of MG in about two-third of patients will develop to severe level within one year. 20\% of MG patients will develop MG crisis within 1 year. MG signs and symptoms will be aggravated in certain conditions such as upper respiratory tract infection, diarrhea, thyroid disease, pregnancy, fever, trauma and medications affect the neuromuscular junctions.

Before the prevalent use of immunosuppressants for MG treatment, the mortality rate of MG is $30 \%$. With also the development of mechanical ventilation and intensive care technique, nowadays the mortality (due to directly MG or indirectly other complication) decreases to below 5\%.

The Chinese version of this guideline has been published in Chin $J$ Neuroimmunol Neurol 2011;18:368-72.

Financial support and sponsorship

Nil.

Conflicts of interest

There are no conflicts of interest. 


\section{REFERENCES}

1. Thanvi BR, Lo TC. Update on myasthenia gravis. Postgrad Med J 2004;80:690-700.

2. Phillips LH. The epidemiology of myasthenia gravis. Semin Neurol 2004;24:17-20.

3. Peng DT, Xu XH, Yu ZY. Determination study of neostigmine test results. Chin J Neuroimmunol Neurol 2007;14:1-3.

4. Zivkovic SA, Shipe C. Use of repetitive nerve stimulation in the evaluation of neuromuscular junction disorders. Am J Electroneurodiagnostic Technol 2005;45:248-261.

5. Cui LY, Guan YZ, Wang H, Tang XF. Single fiber electromyography in th e diagnosis of ocular myasthenia gravis: report of 90 cases. Chin Med J (Engl) 2004;117:848-51.

6. Cont-i Fine BM, Milani M, Kaminski HJ. Myasthenia gravis: past, present, and future. $J$ Clin Invest 2006;16:2843-54.

7. Shigemoto K. Myasthenia gravis induced by autoantibodies against MuSK. Acta Myol 2007;26:185-91.

8. Meriggioli MN, Sanders DB. Advances in the diagnosis of neuromuscular junction disorders. Am J Phys Med Rehabil 2005; 84:627-38.

9. Benatar M. A systematic review of diagnostic studies in myasthenia gravis. Neuromuscul Disord 2006;16:459-67.

10. Keesey JC. Clinical evaluation and management of myasthenia gravis. Muscle Nerve 2004;29:484-505.

11. Skeie GO, Apostolski S, Evoli A, Gilhus NE, Illa I, Harms L, Hilton-Jones D, Melms A, Verschuuren J, Horge HW; European Federation of Neurological Societies. Guidelines for treatment of autoimmune neuromuscular transmission disorders. Eur J Neurol 2010;17:893-902.

12. Jani-Acsadi A, Lisak RP. Myasthenia gravis. Curr Treat Options Neurol 2010;12:231-43.

13. Luchanok U, Kaminski HJ. Ocular myasthenia: diagnostic and treatment recommendations and the evidence base. Curr Opin Neurol 2008;21:8-15.

14. Zhang W, Li ZY. Progress of ocular myasthenia gravis. Chin $J$ Neurol 2007;40:564-6.

15. Antonio-Santos AA, Eggen berger ER. Medical treatment options for ocular myasthenia gravis. Curr Opin Ophthalmol 2008;19:46878.

16. Jani-Acsadi A, Lisak RP. Myasthenic crisis: guidelines for prevention and treatment. J Neurol Sci 2007;261:127-33.

17. Chaudhuri A, Behan PO. Myasthenic crisis. QJM 2009;102:97-107.

18. Bershad EM, Feen ES, Suarez JI. Myasthenia gravis crisis. South Med J 2008; 101:63-69.

19. Parr JR, Jayawant S. C hildhood myasthenia: clinical subtypes and practical management. Dev Med Child Neurol 2007;49:629. 35.

20. Evoli A. Acquired myasthenia gravis in childhood. Curr Opin Neurol 2010;23:536-40.

21. Chiang LM, Darras BT, Kang PB. Juvenile myasthenia gravis. Muscle Nerve 2009;39:423-31.

22. Juel VC, Massey JM. Myasthenia gravis. Orphanet $J$ Rare Dis 2007;2:44.

This guidline is compiled by Nueroimmunology Group of Chinese Society of Neurology. The writing group is shown below (in alphabetical order).

Bao-Guo Xiao (Institute of Neurology, Fudan University); Bi-Tao Bo (Tongji Hospital, Tongji Medical College Huazhong University of Science \& Technology);

Cong Gao (The Second Affiliated Hospital of Guangzhou Medical University);

De-Hui Huang (Chinese PLA General Hospital);

Dong-Ning Wei (The 309th Hospital of Chinese People's Libertation Army);

Fu-Dong Shi (Tianjin Medical University General Hospital);

Guang-Zhi Liu (Peking University People's Hospital);
Hai-Feng Li (Cheeloo University School of Medicine);

Hong-Yu Zhou (West China Hospital, Sichuan University);

Hong-Zeng Li (Tangdu Hospital, The Fourth Military Medical University);

Hua Zhang (Health Department, Beijing Hospital);

Hui-Qing Dong (Xuanwu Hospital Capital Medical University); Jia-Wei Wang (Beijing Tongren Hospital, Capital Medical University);

Jin-Cun Wang (Xijing Hospital, The Fourth Military Medical University);

Lan Chu (The Affiliated Hospital of Guizhou Medicine University);

Li Guo (The Second Hospital of Hebei Medical University);

Li-Hua Wang (The 2nd Affiliated Hospital of Harbin Medical University);

Man-Xia Wang (Lanzhou University Second Hospital);

Mei-Ni Zhang (First Hospital of Shanxi Medical University);

Qi Cheng (Institute of Neurology, Shanghai Jiao Tong University School of Medicine);

Rui-Sheng Duan (Qianfoshan Hospital, Shandong University); Shu-You Fang (The First Affiliated Hospital of Zhenzhou University);

Wei Qiu (The First Affiliated Hospital, Sun Yat-sen University); Wei-Bin Liu (The First Affiliated Hospital, Sun Yat-sen University);

Wei-Ping Wu (Chinese PLA General Hospital);

Wei-Zhi Wang (The 2nd Affiliated Hospital of Harbin Medical University);

Wen-Bin Zhou (Xiangya Hospital Central South University); Xi-De Hou (The First Affiliated Hospital with Nanjing Medical University);

Xiao-Jun Zhang (Beijing Tongren Hospital, Capital Medical University);

Xiao-Kun Qi (Navy General Hospital);

Xian-Hao Xu (Health Department, Beijing Hospital);

Xiang-Jun Chen (Huashan Hospital, Fudan University);

Xiao-Mu Wu (Jiangxi Provincial People's Hospital);

Xiao-Ping Liao (Hainan Medical University);

Xin-Yue Qin (The Hospital Group of the First Affiliated Hospital of CQMU);

Xing-Hu Zhang (Beijing Tian Tan Hospital, Capital Medical University);

Xu Zhang (The First Affiliated Hospital of Wenzhou Medical University);

Xue-An Mo (Institute of Neurology, Guangxi Medical University);

Xue-Qiang $\mathrm{Hu}$ (The Third Affiliated Hospital, Sun Yat-Sen University);

Yan Xu (Peking Union Medical College Hospital);

Yan-Hui Du (General Hospital of Ningxia Medical University); Yang-Tai Guan (Changhai Hospital, The Fourth Military Medical University);

Yu-Wu Zhao (The sixth Affliated Renming Hospital, Shanghai Jiao Tong University School of Medicine);

Ze-Yu Li (Affiliated Hospital of Inner Mongolia Medical College);

Zhong-Ping An (Tianjin Huanhu Hospital);

Zhu-Yi Li (Tangdu Hospital, The Fourth Military Medical University). 